\title{
Erratum to: Image Search with Selective Match Kernels: Aggregation Across Single and Multiple Images
}

\author{
Giorgos Tolias $^{1}$ - Yannis Avrithis ${ }^{2}$ - Hervé Jégou ${ }^{1}$
}

Published online: 12 July 2015

(C) Springer Science+Business Media New York 2015

\section{Erratum to: Int J Comput Vis}

\section{DOI 10.1007/s11263-015-0810-4}

During the publication process, an error was introduced regarding Table 3. The corrected table appears below:

Table 3 Performance evaluation for different feature set sizes, extracted by using different detector threshold values

\begin{tabular}{|c|c|c|c|c|c|c|c|c|c|c|c|c|}
\hline \multirow{3}{*}{$\begin{array}{l}\text { Dataset } \\
\text { Method }\end{array}$} & \multicolumn{4}{|c|}{ Oxford5k } & \multicolumn{4}{|l|}{ Paris6k } & \multicolumn{4}{|c|}{ Holidays } \\
\hline & \multicolumn{2}{|l|}{ Small } & \multicolumn{2}{|l|}{ Large } & \multicolumn{2}{|l|}{ Small } & \multicolumn{2}{|l|}{ Large } & \multicolumn{2}{|l|}{ Small } & \multicolumn{2}{|l|}{ Large } \\
\hline & SMK & ASMK & SMK & ASMK & SMK & ASMK & SMK & ASMK & SMK & ASMK & SMK & ASMK \\
\hline SA & 74.9 & 78.1 & 78.5 & 82.0 & 70.9 & 76.0 & 73.2 & 78.7 & 78.6 & 81.2 & 84.0 & 88.0 \\
\hline MA & 77.4 & 81.7 & 79.3 & 83.8 & 71.8 & 78.2 & 74.2 & 80.5 & 79.0 & 82.2 & 82.9 & 86.5 \\
\hline Method & $\mathrm{SMK}^{\star}$ & $\mathrm{ASMK}^{\star}$ & $\mathrm{SMK}^{\star}$ & $\mathrm{ASMK}^{\star}$ & $\mathrm{SMK}^{\star}$ & $\mathrm{ASMK}^{\star}$ & $\mathrm{SMK}^{\star}$ & $\mathrm{ASMK}^{\star}$ & $\mathrm{SMK}^{\star}$ & $\mathrm{ASMK}^{\star}$ & $\mathrm{SMK}^{\star}$ & $\mathrm{ASMK}^{\star}$ \\
\hline SA & 73.7 & 76.4 & 77.5 & 80.3 & 69.8 & 74.4 & 72.0 & 77.2 & 77.7 & 80.0 & 83.1 & 86.5 \\
\hline MA & 77.4 & 80.4 & 78.2 & 82.7 & 70.9 & 77.0 & 73.0 & 79.3 & 77.8 & 81.1 & 81.0 & 84.4 \\
\hline \# Features & $12.5 \mathrm{M}$ & $11.2 \mathrm{M}$ & $21.9 \mathrm{M}$ & $19.2 \mathrm{M}$ & $15.0 \mathrm{M}$ & $13.0 \mathrm{M}$ & $25.1 \mathrm{M}$ & $21.5 \mathrm{M}$ & $4.4 \mathrm{M}$ & $3.5 \mathrm{M}$ & $16.7 \mathrm{M}$ & $12.0 \mathrm{M}$ \\
\hline
\end{tabular}

Small set with the default threshold, large set with lower threshold. Number of features indexed without (SMK-SMK $\left.{ }^{\star}\right)$ and with $\left(\right.$ ASMK-ASMK $\left.{ }^{\star}\right)$ aggregation are reported. Performance for single (SA) and multiple (MA) assignment. These results are without spatial verification and without QE. Bold values corresponds to the best score per dataset

The online version of the original article can be found under doi:10.1007/s11263-015-0810-4.

\footnotetext{
$凶$ Giorgos Tolias

giorgos.tolias@inria.fr

1 INRIA, Rennes, France

2 NTUA, Athens, Greece
} 